\title{
Flexible, Front-Facing Luminescent Solar Concentrators Fabricated from Lumogen F Red 305 and Polydimethylsiloxane
}

\author{
Ian A. Carbone $\mathbb{D}^{1}{ }^{1}$ Katelynn R. Frawley, ${ }^{1}$ and Melissa K. McCann ${ }^{2}$ \\ ${ }^{1}$ Department of Environmental Science and Sustainability, Allegheny College, Meadville, PA 16335, USA \\ ${ }^{2}$ Department of Materials Science and Engineering, Washington University, St. Louis, MO 63160, USA \\ Correspondence should be addressed to Ian A. Carbone; icarbone@allegheny.edu
}

Received 24 January 2019; Revised 12 March 2019; Accepted 28 March 2019; Published 30 April 2019

Guest Editor: Mattia De Rosa

Copyright (C) 2019 Ian A. Carbone et al. This is an open access article distributed under the Creative Commons Attribution License, which permits unrestricted use, distribution, and reproduction in any medium, provided the original work is properly cited.

\begin{abstract}
Luminescent solar concentrators (LSCs) fabricated with transparent host materials and fluorescent organic dyes are cost effective and versatile tools for solar power production. In this study, the first flexible, front-facing LSCs utilizing Lumogen F Red 305 (LR305) and polydimethylsiloxane (PDMS) were demonstrated. Bulk-doped devices, fabricated with dye evenly distributed throughout the waveguide, were optimized for light gain with LR305 concentrations between 0.075 and $0.175 \mathrm{~g} / \mathrm{l}$. Thin-film devices, fabricated with a thin layer of luminescent material applied to the bottom side of the waveguide, were optimized between 0.5 and $0.75 \mathrm{~g} / \mathrm{l}$. The bulk-doped and thin-film devices produced light gains of 1.86 and 1.89 , respectively, demonstrating that flexible designs can be developed without sacrificing power production. Bulk-doped devices proved to be less effective than thin-film devices at collecting direct light due to the placement of fluorescent dyes above the front-facing solar cell. Thin-film devices demonstrated less light collection than bulk-doped devices further from the device centers possibly due to quenching and self-absorption losses at higher dye concentrations. Light collection was minimally impacted by moderate bending in both LSC designs, suggesting that flexible, front-facing devices could be effectively deployed on curved and uneven surfaces. Finally, optical measurements of the LSC waveguides suggest that they could support plant growth underneath. Similar designs could be developed for applications in agricultural settings.
\end{abstract}

\section{Introduction}

Photovoltaic module prices have decreased dramatically over the last decade $[1,2]$, and by some estimates, U.S. and global solar capacities will more than double in the next five years $[3,4]$. In light of the growth and trajectory of solar markets, it is important to investigate the space-related requirements and limitations of solar technologies in order to develop land resources mindfully. Several researchers have explored best practices for minimizing land use and environmental disturbances when working with more established solar technologies [5-9], while several others have developed systems that allow for power generation in a greater variety of locations [10-14]. Luminescent solar concentrators (LSCs), first developed in the 1970s, are a promising group of semitransparent solar devices that can be deployed in a variety of alternative settings [15-17]. In this manuscript, we explore the use of
LSCs for more efficient solar power generation and space utilization in agricultural settings.

LSCs consist of one or more solar cells attached to a transparent plate, or waveguide, that has been doped with a fluorescent dye [16, 17]. Dye molecules can be dispersed throughout the waveguiding material in what we refer to as a "bulk-doped" device or applied in a concentrated layer to the front or back of what is commonly called a "thin-film" device [18]. In both cases, the fluorescent material serves to absorb and reemit light inside the waveguide, where a significant fraction becomes trapped due to total internal reflection. Strategically placed solar cells mounted onto the waveguides collect concentrated light and generate electricity. Due to their semitransparent designs, LSCs can be developed for a greater variety of applications than conventional photovoltaics. Waveguides can be designed to absorb and transmit desired portions of the electromagnetic spectrum, 


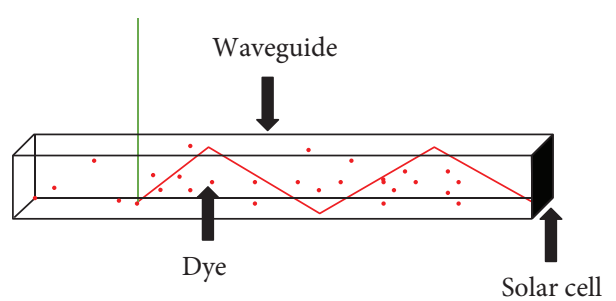

(a)

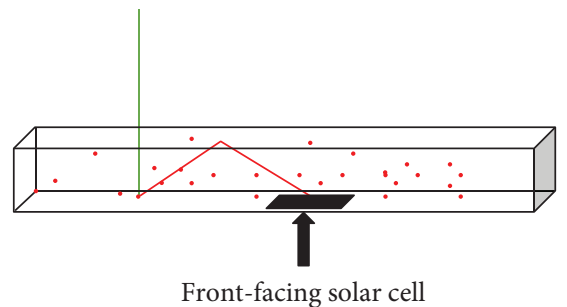

(c)

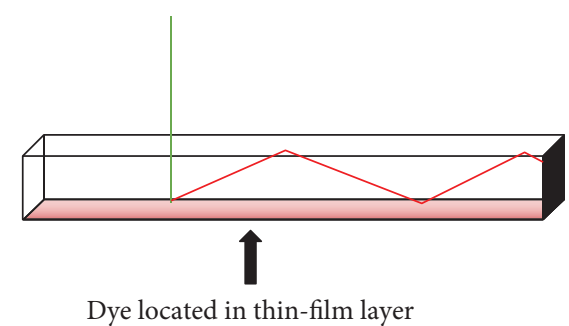

(b)

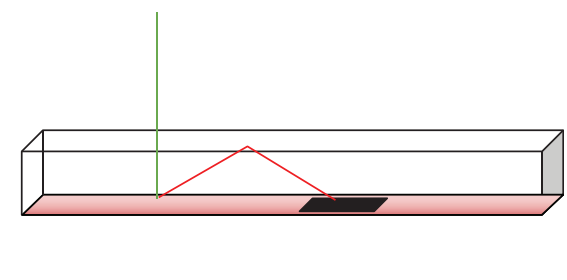

(d)

FIgUre 1: Conventional bulk-doped (a) and thin-film (b) LSC designs utilizing edge-mounted solar cells. Front-facing bulk-doped (c) and thin-film (d) LSC designs. Dye molecules are dispersed throughout the waveguiding material in the bulk-doped device and applied in a concentrated layer to the backside of the thin-film device. Both designs rely on total internal reflection in the waveguide to concentrate light.

and LSC panels can be integrated into built environments as power-generating windows $[14,19-23]$.

Most published LSC designs place solar cells on the edges of waveguides in order to maximize light gain (Figures 1(a) and 1(b)); however, some researchers have focused recent efforts on developing bottom-mounted, or front-facing, designs (Figures 1(c) and 1(d)) [24-30]. By orienting the solar cells to face the light source, they are able to collect direct light in addition to concentrated light. On larger LSCs with significant distances between edges, front-facing cells can also be strategically placed to minimize the average distance traveled by concentrated light in the waveguide. Another advantage of front-facing designs is that they allow designers to control the fraction of the LSC area that is covered by solar cells. Front-facing LSCs with power conversion efficiencies of $6.8 \%$ have been fabricated by covering $31 \%$ of the device area with solar cells [24]. Other devices have been able to double the photon flux received by embedded solar cells with $5 \%$ solar cell coverage [24].

In addition to device geometry, dye selection and processing can significantly impact LSC performance. Dye-dye and dye-host interactions can cause quenching and/or selfabsorption losses, and waveguides often decrease in performance when dyes are highly concentrated or unevenly distributed in their host matrix [31-33].

BASF's Lumogen F Red 305 (LR305) is a soluble perylene dye with a high fluorescence quantum yield and high photostability, making it one of the most popular LSC dyes available [31-33]. LR305 is also a well-suited LSC material for agricultural applications. When LR305 is suspended in ethanol, polycarbonate, and polydimethylsiloxane (PDMS), absorbance maxima occur at 578, 573, and $560 \mathrm{~nm}$, respectively, and emission maxima in these materials occur at 613,597 , and $590 \mathrm{~nm}$, respectively [31]. Because most plants rely on the red and blue portions of the solar spectrum,
LR305-based materials can avoid interfering with the wavelengths required for photosynthesis. In 2016, Corrado et al. [19] demonstrated vegetable production underneath frontfacing LSC panels fabricated with LR305 in a demonstration greenhouse. A follow-up study concluded that these greenhouse LSCs had no negative impacts on tomato production and that some varieties of crops might respond positively to the LSC greenhouse conditions [34]. Similar greenhouse panels that offer wavelength-selective power production have since entered the marketplace, and there appears to be a growing market for solar materials that also support growing environments $[35,36]$. As LSCs continue to be developed for agricultural applications, there is an opportunity to develop new devices that can be deployed in growing environments that are ill-suited for rigid LSC panels. For curved and uneven surfaces, like hoop houses, low tunnels, and open fields, a flexible LSC fabric would be far more easily deployed and maintained.

A number of studies have developed flexible LSCs $[28,31,37-41]$, and one of the most well-documented flexible waveguide materials is polydimethylsiloxane (PDMS), a silicon-based organic polymer with high transparency, flexibility, and durability. Past research has demonstrated successful edge-mounted LSCs using PDMS and LR305 $[31,42]$; however, this study is the first to utilize these materials in a front-facing LSC design. After optimizing bulk-doped and thin-film designs for dye concentration, a detailed analysis of waveguide properties and light gains is carried out to help guide future device development.

\section{Materials and Methods}

2.1. Device Fabrication. To begin the LSC fabrication process, reference cells were prepared and characterized before being incorporated into finished LSC devices. $17.5 \%$ 


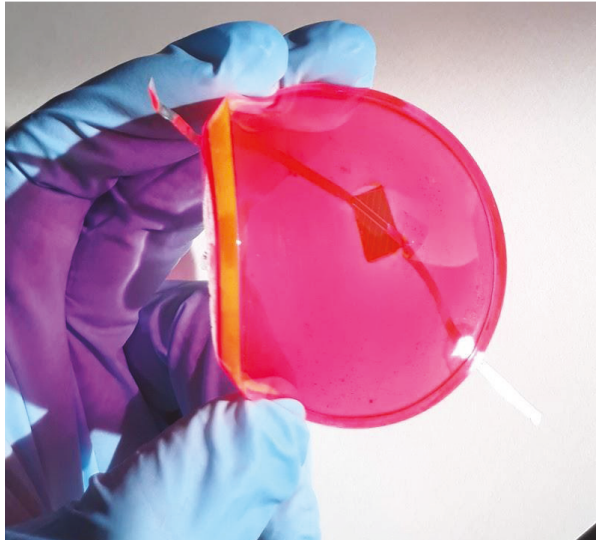

(a)

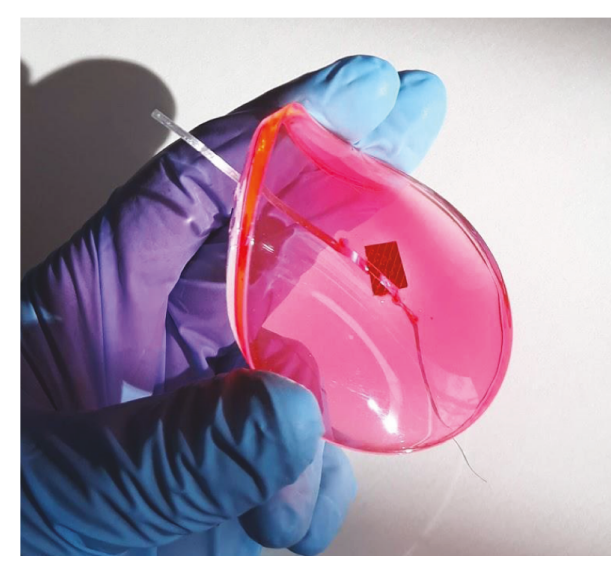

(b)

FIGURE 2: Finished LSC devices. The bulk-doped LSC (a) has dye evenly distributed throughout the waveguide. The thin-film LSC (b) has a thin $(\sim 0.26 \mathrm{~mm})$ layer of concentrated dye applied to the bottom surface. The front-facing solar cell can be seen in the center of both flexible devices.

efficient MISOL monocrystalline solar cells were cut into $\sim 1 \mathrm{~cm} \times 1 \mathrm{~cm}$ reference cells using a diamond scribe, and 3 inches of tabbing wire were soldered onto the front and back contacts. More precise reference cell areas were measured using a HP Scanjet 8300 scanner and GIMP image manipulation software for the calculation of reference cell efficiencies. Finally, reference cells were electrically characterized (see details in Section 2.3) and set aside until the waveguides were fabricated.

Bulk-doped waveguides were constructed by combining Dow Corning SYLGARD 184 base and curing agents with a base to curing agent ratio of $10: 1$. BASF LR305, which was stored in an $8 \mathrm{~g} / \mathrm{l}$ toluene solution, was combined with the SYLGARD 184 mixtures to achieve the desired device dye concentrations. After combining thoroughly by hand, the PDMS mixtures were poured into $7 \mathrm{~cm}$ diameter glass petri dishes to form $4 \mathrm{~mm}$ thick films. The PDMS mixtures were placed into an evacuated vacuum oven until all air bubbles were released and then heated for 30 minutes at $100^{\circ} \mathrm{C}$ to cure. After removing the solid waveguides from the petri dishes, reference cells were attached to the centers of the waveguides using a small amount of the undoped SYLGARD 184 mixture. The materials were heated for an additional 10 minutes to complete the bulk-doped LSCs. We found that the durability of finished LSCs could be significantly increased by attaching a thin layer of rigid material to the back of the solar cells; however, this was not necessary for the devices characterized in this study. A finished bulkdoped device is pictured in Figure 2(a).

The thin-film device fabrication process began by modifying the bulk-doped procedure to produce clear waveguides that contained no dye. After attaching reference cells to the clear waveguides, SYLGARD 184 mixtures were created with the desired thin-film dye concentrations. $1 \mathrm{ml}$ of the dye mixtures was poured onto the back of each waveguide, spread with a spatula, and allowed to settle for one hour on a level surface. The devices were heated for an additional 10 minutes at $100^{\circ} \mathrm{C}$ to cure the dye-containing layers. A finished thinfilm device is pictured in Figure 2(b).
It is worth recognizing that our "thin-film" LSCs belong to a broader class of designs that contain concentrated layers of dye on the top or bottom surfaces of the waveguide. The dye-containing layers used in this study, however, would not be considered "thin-films" by the standards of modern materials science. Our fabrication methods deposited films that were approximately $0.26 \mathrm{~mm}$ thick.

In this experiment, bulk-doped and thin-film LSCs were fabricated and tested with concentrations ranging from 0.02 to $0.50 \mathrm{~g} / \mathrm{l}$ and 0.25 to $1.00 \mathrm{~g} / \mathrm{l}$, respectively. 3 LSCs were constructed at 7 concentrations for each device type. The 42 LSCs considered in this study were optically and electrically characterized as described in the sections below.

2.2. Optical Characterization. Absorbance and transmittance measurements of LSC waveguides were carried out on an Ocean Optics spectroscopy system with USB2000+ spectrometer, LS-1 light source, and SpectraSuite software. Absorbance spectra of bulk-doped and thin-film LSCs allow us to compare light collection in the two designs. Transmittance values are compared to the absorbance spectra of plant pigments to assess LSC compatibility with growing environments. For reference, the optical properties of LR305-doped PDMS waveguides have been studied in greater detail by Buffa et al. [31] and Steinbruck et al. [43].

2.3. Electrical Characterization. I-V curves of naked reference cells and finished LSCs were measured with an AM1.5 AbetTechnologies SunLite 11002 solar simulator and Keithley 2400 SourceMeter. During characterization, the LSCs were situated above a sheet of $83 \%$ reflective office paper by placing $1.6 \mathrm{~mm}$ foam spacers underneath the center and outer edges of the LSCs. A $4 \mathrm{~mm}$ strip of $83 \%$ reflective paper was also taped around the outer edges of the devices to standardize test conditions across devices and measurements. Short circuit currents, open circuit voltages, fill factors, and power conversion efficiencies were calculated from the I-V curves of each LSC before and after the attachment of the reference cells to quantify changes in light collection. The 


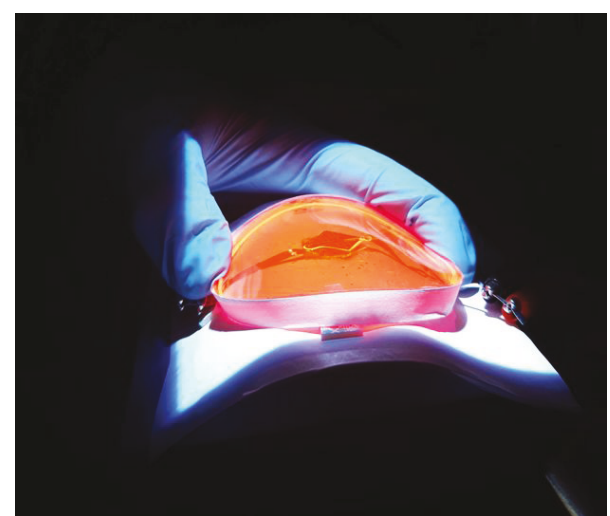

FIgURE 3: Electrical characterization of a bulk-doped LSC device pressed over a $5 \mathrm{~cm}$ bend tool. The LSC is placed above and inside of the $83 \%$ reflective paper. Due to the rigid solar cell placed in the device center, only regions outside the $1 \mathrm{~cm} \times 1 \mathrm{~cm}$ square were allowed to bend.

majority of this study's analysis focuses on changes to the short circuit current, which are directly proportional to changes in the photon flux received by the reference cells. We define light gain as follows:

$$
\text { Light gain }=\frac{I_{\text {LSC-short circuit }}}{I_{\text {Reference cell-short circuit }}} .
$$

After the initial device characterization, a masking experiment was carried out to compare light collection from different locations on the LSC surfaces. Masks were created to shade the perimeter regions of each device from incident light, and light gains were measured over a range of mask radii. Light gains were then divided by the exposed areas, and values were compared in order to highlight differences between the inner and outer regions of the LSC devices.

Bent devices were also electrically characterized to investigate the impacts of bending on light concentration. Like the above measurements, LSCs were placed $1.6 \mathrm{~mm}$ above $83 \%$ reflective paper. Both the paper and the LSCs were pressed over bend tools (partial cylinders with radii of curvature between 2 and $8 \mathrm{~cm}$ ) and characterized with I-V curves. For clarity, Figure 3 provides an image of a bulk-doped LSC being characterized over a $5 \mathrm{~cm}$ bend tool.

Device performances during the bending experiments were calculated according to

$$
\text { Bent performance }=\frac{I_{\text {Bent LSC }}-I_{\text {Ref.cell }}}{I_{\text {Flat LSC }}-I_{\text {Ref. cell }}} .
$$

Following this equation, short circuit current measurements of the naked reference cells were subtracted from the short circuit currents of completed, bent LSCs in order to isolate light gains attributed to concentrated light in the waveguide (as opposed to the collection of direct light). These values were then scaled by their unbent values so that trends could be easily compared across devices.

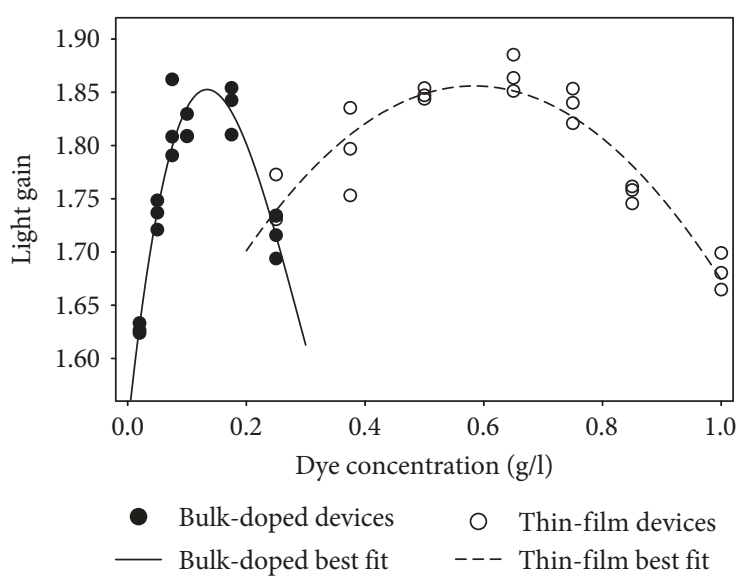

FIgURE 4: Light gains of bulk-doped and thin-film devices plotted against dye concentration. Bulk-doped devices were optimized for light gain with LR305 concentrations between 0.075 and $0.175 \mathrm{~g} / \mathrm{l}$. Thin-film devices were optimized between 0.5 and $0.75 \mathrm{~g} / \mathrm{l}$. Best fit second-order polynomials are included to visualize trends in each data set.

\section{Results and Discussion}

3.1. Optimization of Dye Concentrations. Both bulk-doped and thin-film LSCs were fabricated over a range of dye concentrations and characterized so that light gains could be compared. Figure 3 presents the light gains of all 42 LSCs considered in this study and shows that individual devices varied in performance by up to $5 \%$ even when they utilized the same design and dye concentration. These differences were likely due to minor variations in device construction. Bonds between device layers and dye distributions varied slightly between LSCs as did the sizes and placements of solar cells and contacts.

Despite these differences, the light gains in both sets of devices were clearly correlated with dye concentration. Figure 4 indicates that bulk-doped devices were optimized with LR305 concentrations between 0.075 and $0.175 \mathrm{~g} / \mathrm{l}$. These values are consistent with the optimized concentrations found by Buffa et al. [31] for side-mounted LSCs made from LR305 and PDMS.

Because thin-film devices utilized thinner layers of luminescent material, higher dye concentrations were necessary to maintain light absorption. The thin-film LSCs in this study exhibited their highest light gains between 0.5 and $0.75 \mathrm{~g} / \mathrm{l}$, which is significantly lower than previously published thin-film LSCs made with LR305 and PMMA [33, 44]. Dye aggregation issues documented in previous studies of LR305 and PDMS may be responsible for limiting performance at higher concentrations [31].

3.2. Individual Devices. The individual bulk-doped and thinfilm LSCs with the largest light gains are characterized in greater detail below. The IV curves of reference cells and completed LSCs presented in Figure 5 allowed for the calculations of the efficiencies and light gains presented in Table 1.While the thin-film device with $0.65 \mathrm{~g} / \mathrm{l}$ dye 


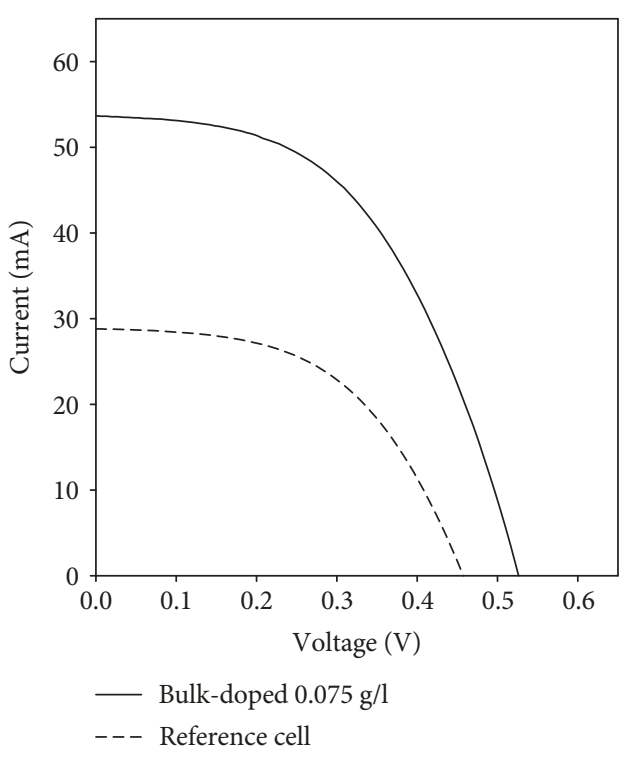

(a)

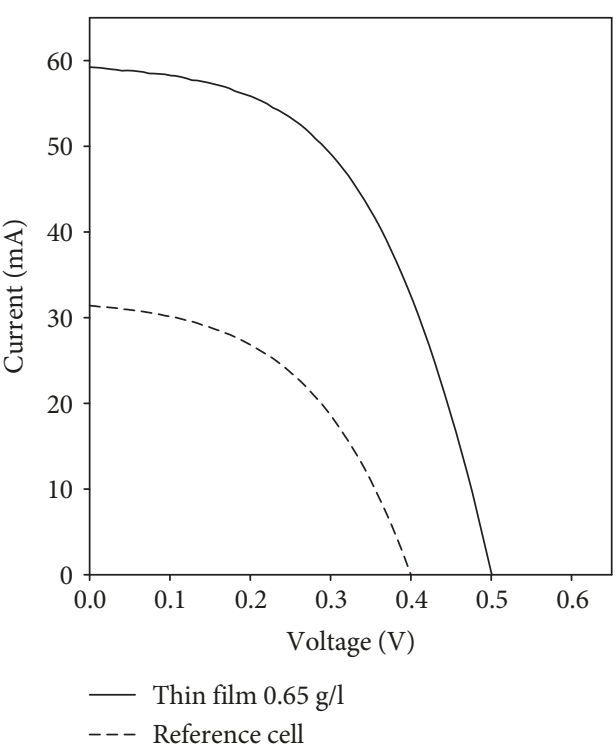

(b)

FIGURE 5: IV curves of optimized bulk-doped (a) and thin-film (b) LSCs and reference cells. Open circuit voltages and short circuit current both experience gains with increased irradiance. The relatively low open circuit voltages of the naked reference cells may suggest that a relatively high fraction of incident light was obstructed by contacts and tabbing wire located on the cell surface.

TABLE 1: Characteristics of optimized bulk-doped and thin-film LSCs.

\begin{tabular}{lcccc}
\hline Device structure & Dye concentration $(\mathrm{g} / \mathrm{l})$ & Light gain & Ref. cell efficiency (\%) & Final device efficiency (\%) \\
\hline Bulk-doped & 0.075 & 1.86 & 6.43 & 0.37 \\
Thin film & 0.65 & 1.89 & 5.76 & 0.39 \\
\hline
\end{tabular}

concentration recorded a slightly greater light gain than the $0.075 \mathrm{~g} / \mathrm{l}$ bulk-doped device, the two values were within the ranges of experimental uncertainty observed between similarly constructed devices. In an earlier study of thin-film, front-facing devices made with LR305 and PMMA, Corrado et al. [24] demonstrated a light gain of 1.85 for LSCs with the same cell size $(1 \mathrm{~cm} \times 1 \mathrm{~cm})$ and coverage $(2.6 \%)$ as the devices in this study. This value is $3 \%$ less than the optimized light gains in this study, suggesting that flexible devices can be constructed with PDMS as opposed to PMMA without sacrificing light concentration.

The efficiencies of optimized bulk-doped and thin-film LSCs were $0.37 \%$ and $0.39 \%$, respectively. These values are lower than many previously published front-facing LSCs $[24,29,30]$ for two major reasons. First, the LSCs in this study were designed to optimize light gain rather than device efficiency. As a result, a relatively small fraction (2.6\%) of the LSC areas were covered with solar cells, resulting in lower efficiencies. Secondly, the hand-cut and soldered reference cells had relatively low efficiencies before they were incorporated into completed LSCs. The relatively low open circuit voltages of the naked reference cells in Figure 5 suggest that a relatively high fraction of incident light was obstructed by contacts and tabbing wire located on the cell surface. While this should not have had a significant impact on light gain measurements and calculations, it did negatively impact on overall LSC efficiency. If future devices could achieve the same light gains using record-holding $26.7 \%$ efficient monocrystalline cells, these LSC designs could theoretically achieve efficiencies of $1.29 \%$ for bulk-doped devices and $1.31 \%$ for thin-film devices [45].

3.3. Waveguide Absorption. Bulk-doped and thin-film LSCs produced similar light gains, but the two designs exhibited important differences in their optical properties. If the dye properties and light collection processes remained consistent between devices, we would expect the thin-film and bulkdoped LSCs to be optimized with similar optical densities and similar dye quantities (even if the dye might be more concentrated in the thin-film construction) [46]. However, the optimized thin-film LSC contained 58\% less dye than the optimized bulk-doped device, and absorbance values in Figure 6 demonstrate that the thin-film LSC exhibited lower absorbance values across the spectrum. While both waveguides demonstrate an absorbance peak at $565 \mathrm{~nm}$, the thin-film absorbance in this region is almost $56 \%$ less than the bulk-doped measurement. One possible explanation for the low optical densities of optimized thin-film LSCs is that dye interactions were limiting device performance at higher concentrations.

These results are supported by studies that have characterized LR305-doped PDMS in greater detail. Steinbruck 


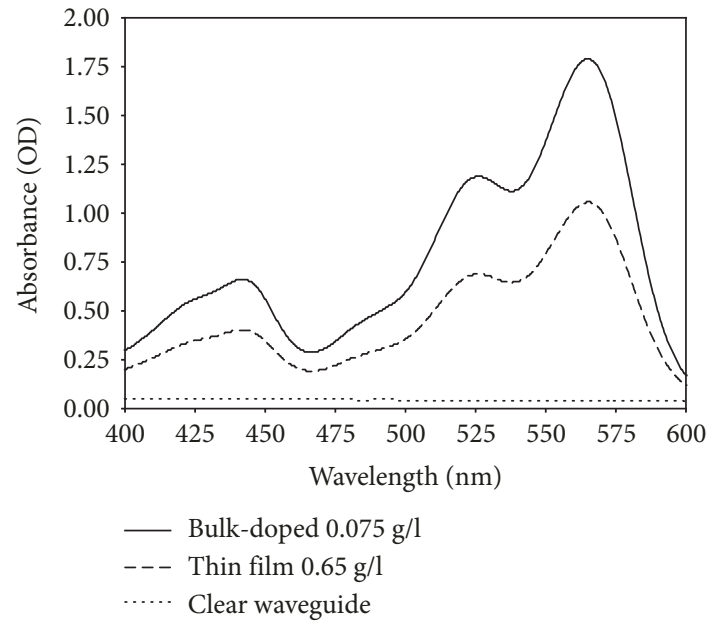

FIGURE 6: Absorbance spectra of bulk-doped, thin-film, and clear LSC waveguides. The thin-film waveguide had a 56\% lower absorbance than the bulk-doped waveguide at $565 \mathrm{~nm}$. Quenching and self-absorption may limit thin-film device performance at higher dye concentrations, resulting in devices that are optimized with lower optical densities.

et al. [43] found that LR305 is prone to aggregation in PDMS due to low solubility. Similarly, Buffa et al. [31] observed quenching and self-absorption losses in PDMS waveguides when concentrations of LR305 exceeded $0.1 \mathrm{~g} / \mathrm{l}$. The optimized thin-film LSC in this study had a concentration of $0.65 \mathrm{~g} / \mathrm{l}$ (well above $0.1 \mathrm{~g} / \mathrm{l}$ ) suggesting that thin-film devices were limited to lower optical densities than the bulk-doped LSCs due to increased quenching and self-absorption losses at higher dye concentrations.

3.4. Light Gain and Aperture. To compare light collection from the inner regions (near the device center) and outer regions (near the device perimeter) of the finished LSCs, light gains were measured while the device perimeters were shaded from incident light. Figure 7 presents the light gains per unit of exposed area for the optimized bulk-doped and thin-film LSCs. Bulk-doped and thin-film devices both exhibited similar trends to previously characterized rigid devices $[24,29,30]$. Light gain contributions were greatest near the device centers due to the capture of direct light and the short travelling distance for concentrated light. At locations closer to the device perimeters, the light gain contributions were diminished due to dye and waveguide reabsorption.

The thin-film device in Figure 7 outperformed the bulkdoped device at smaller apertures due to differences in the waveguide properties above the solar cell. Unlike the thinfilm device, the bulk-doped solar cell was mounted beneath a waveguide that contained dye, and this dye absorbed and reemitted some direct light before it could be collected by the solar cell. Because the bulk-doped and thin-film devices performed similarly when most or all of the device surfaces were exposed to incoming light, we conclude that the bulkdoped device was more effective at collecting concentrated light from the outer regions of the waveguide. Poor light harvesting from the thin-film waveguide is consistent with the

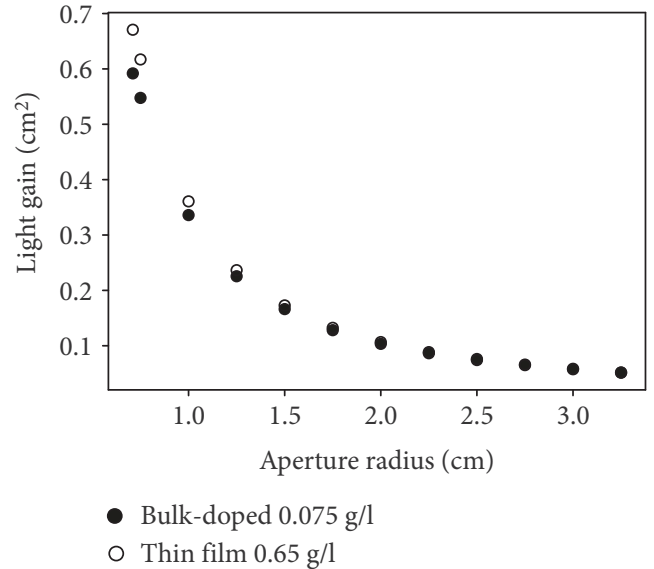

FIGURE 7: LSC light gains per unit exposed area during masking experiments. The thin-film device was more effective at collecting direct light. The bulk-doped device was more effective at collecting concentrated light from the waveguide.

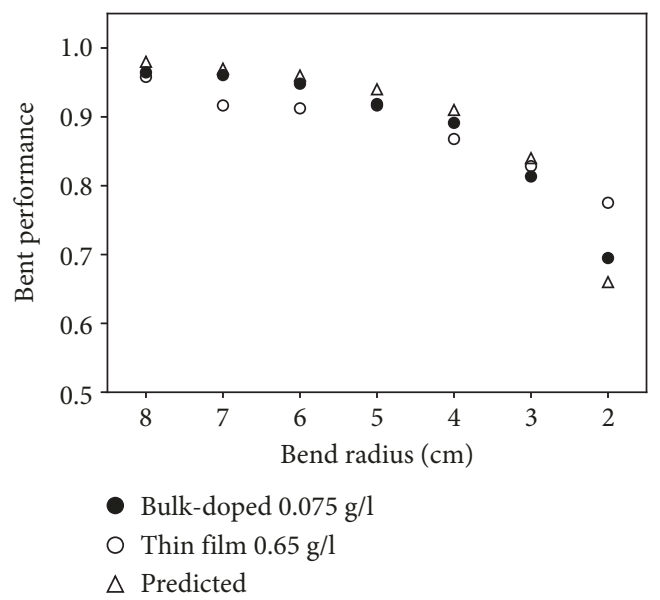

Figure 8: Bent waveguide performance. Values represent the bent waveguide light gains scaled by the flat waveguide light gains. Both devices performed similarly to predicted values determined by the fraction of light intercepted by the bent geometry.

lower waveguide absorbance and possible dye interactions found in Section 3.3.

3.5. Device Bending. Bent devices were characterized to explore the feasibility of deploying front-facing LSCs on curved and/or uneven surfaces. Figure 8 shows the bent waveguide performance when the optimized devices were curved over bend tools with radii between 8 and $2 \mathrm{~cm}$. To quantify our bend performance, short circuit currents of device reference cells were subtracted from the short circuit currents of the finished devices and the results were scaled by the corresponding unbent values (see equation (2)).

Figure 8 indicates that the optimized thin-film LSC was less impacted than the bulk-doped devices at small bend radii. This result is consistent with the masking results 


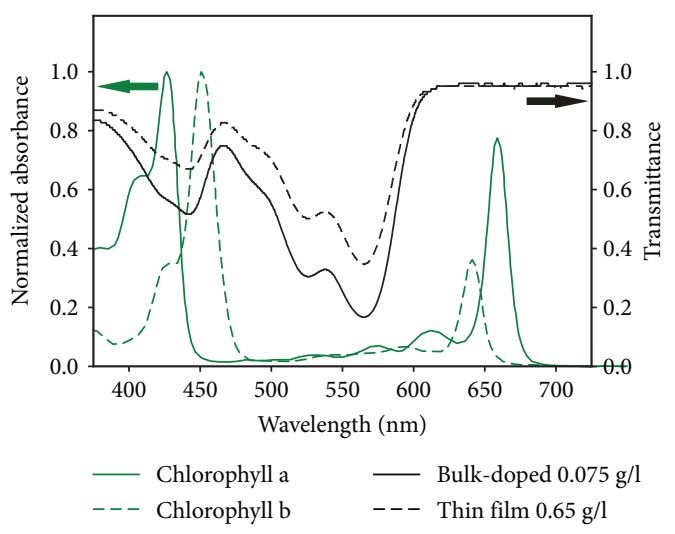

FIgURE 9: Waveguide transmittance and normalized absorbance of chlorophylls $\mathrm{a}$ and $\mathrm{b}$ in diethyl ether. Waveguides of both devices primarily concentrate wavelengths between 500 and $600 \mathrm{~nm}$ and transmit the majority of the light absorbed by the two primary plant pigments. The optimized thin-film LSC transmits more light due to its lower optical density. Absorbance measurements were taken from Lichtenthaler [47].

discussed in Section 3.4. More extreme bending disproportionately impacted light collection near the device perimeters where reabsorption losses had their greatest impact. The thin-film device was less impacted by this bending due to the losses that were already occurring near the LSC perimeter.

In a study on flexible LSCs made with monocrystalline $\mu$-cells, Yoon et al. [28] found that light gains in bent devices were proportional to the areas of the bent devices projected onto a plane normal to the incident light. To test devices in this study against this trend, values for projected device areas were expressed as fraction of flat device areas and included in Figure 8. The LSCs in this study followed the predicted trend with the exception of the thin-film device at the $2 \mathrm{~cm}$ bend radius. The general agreement with the predicted values for both LSCs is consistent with findings from Tummeltshammer et al. [41] who showed that the moderate bending of flexible waveguides does not significantly increase escape cone losses.

3.6. Growing Environment. Optical measurements of our optimized waveguides provide insight into the agricultural growing environments that could be supported underneath the LSC materials considered in this study. Figure 9 presents waveguide transmittance values alongside the absorbance spectra of chlorophylls a and $\mathrm{b}$, the primary photoreceptor pigments in photosynthesis. Absorbance spectra, taken from Lichtenthaler [47], demonstrate that chlorophyll a absorbs most strongly at $428 \mathrm{~nm}$ and $660 \mathrm{~nm}$ and chlorophyll b absorbs most strongly at $452.2 \mathrm{~nm}$ and $641.8 \mathrm{~nm}$. All of these values are distinct from the minimum waveguide transmittance at $565 \mathrm{~nm}$.

More than $95 \%$ of photosynthetically active light in the red portion of the solar spectrum was transmitted through the optimized LSC waveguides. However, transmittance was significantly diminished at photosynthetically active blue wavelengths due to the broad shoulder of the LR305 absorption peak. The transmittance of the optimized bulkdoped waveguide at $426 \mathrm{~nm}$, for example, was only $57 \%$. The thin-film waveguide transmitted a larger fraction of these blue wavelengths, due to its lower dye content and optical density. Because the thin-film design both absorbed less light than the bulk-doped design and emitted less downshifted light from the surfaces of the waveguide, the two waveguides exhibited similar transmittance values in the red portion of the spectrum.

Despite the altered spectrum created by the LR305PDMS layers, this LSC technology still holds promise for applications in agricultural operations. The impacts of these waveguides on incident spectra are nearly identical to those documented in LR305-PMMA/glass greenhouse panels that successfully supported tomato production in past studies $[19,34]$. Researchers in one study also noted that crop varieties have varied responses to altered spectra and some varieties may benefit from reduced levels of blue light [34]. The best designs for future growing conditions will almost certainly depend on the needs of the specific plant varieties being grown.

\section{Conclusions}

In this study, the first flexible front-facing LSCs utilizing LR305 and PDMS were demonstrated. Bulk-doped devices were optimized for light gain with LR305 concentrations between 0.075 and $0.175 \mathrm{~g} / \mathrm{l}$. Thin-film devices were optimized between 0.5 and $0.75 \mathrm{~g} / \mathrm{l}$. The two device constructions demonstrated similar light gains to comparable rigid devices made with PMMA and LR305. Bulk-doped devices proved to be less effective at collecting direct light, and thin-film devices appeared less effective at concentrating light due to quenching and self-absorption losses at higher dye concentrations. Light collection was minimally impacted by moderate bending in both LSC designs, suggesting that flexible, front-facing devices could be developed into effective tools for power generation on curved and uneven surfaces.

Our data suggest that quenching and self-absorption losses are important limiting factors in LR305-PDMS LSCs. Future research should be carried out to quantify escape cone and nonunity quantum yield losses in flexible, front-facing devices. Another area of inquiry is to explore other flexible host materials such as polymethylphenylsiloxane (PMPS) and polyethylene terephthalate (PET).

Transmittance measurements of the optimized waveguides in this study suggest that both designs significantly alter the potential growing conditions underneath the devices. In particular, they significantly reduce the amount of blue light that plants would receive if these technologies were to be installed above crops in agricultural settings. However, past studies on rigid LSC panels suggest these conditions can still support productive growing environments [34]. LSCs modeled after our bulk-doped designs would support growing environments with less blue light than those modeled after our thin-film designs, but the best designs for future growing conditions will depend on the needs of the specific plant varieties being grown. 
Future research is still needed to better understand how specific crop varieties respond to these altered spectra (in particular, reduced levels of blue light). Another area of concern is the long-term durability of LR305-PDMS devices in outdoor environments. For this reason, a variety of UV stabilizers and flexible host materials should be considered and subjected to reliability and durability testing. Additional prototypes should also be developed to optimize device parameters such as durability, waveguide thickness, solar cell size, cell placement, and growing environment.

\section{Data Availability}

The experimental data used to support the findings of this study are included within the supplementary information file(s) (available here).

\section{Conflicts of Interest}

The authors declare that there is no conflict of interest regarding the publication of this paper.

\section{Acknowledgments}

The authors give special thanks to Bruce Wittmershaus, Ph.D., for proof reading this article. Materials and summer researchers that supported this research were paid for by Allegheny College.

\section{Supplementary Materials}

All data presented in this article are contained in the supplementary file titled "Flexible Front-Facing LSC Data.xls." Data for Figures 4-9 are contained on spreadsheets labeled by their respective figures. For more information about this file, feel free to contact the corresponding author. (Supplementary Materials)

\section{References}

[1] IRENA, Renewable Power Generation Costs in 2017, International Renewable Energy Agency, 2018.

[2] G. Kavlak, J. McNerney, and J. E. Trancik, "Evaluating the causes of cost reduction in photovoltaic modules," Energy Policy, vol. 123, pp. 700-710, 2018.

[3] SEIA and Wood Mackenzie Power \& Renewables, U.S. Solar Market Insight, Solar Energy Industries Association, 2018.

[4] IEA, Renewables 2018 - Market Analysis and Forecast from 2018 to 2023, International Energy Agency, 2018.

[5] R. Chiabrando, E. Fabrizio, and G. Garnero, "The territorial and landscape impacts of photovoltaic systems: definition of impacts and assessment of the glare risk," Renewable and Sustainable Energy Reviews, vol. 13, no. 9, pp. 2441-2451, 2009.

[6] R. R. Hernandez, S. B. Easter, M. L. Murphy-Mariscal et al., "Environmental impacts of utility-scale solar energy," Renewable and Sustainable Energy Reviews, vol. 29, pp. 766-779, 2014.

[7] R. Lakhani, G. Doluweera, and J. Bergerson, "Internalizing land use impacts for life cycle cost analysis of energy systems: a case of California's photovoltaic implementation," Applied Energy, vol. 116, pp. 253-259, 2014.

[8] S. Ong, C. Campbell, P. Denholm, R. Margolis, and G. Heath, "Land-use requirements for solar power plants in the United States," Tech. Rep., National Renewable Energy Lab. (NREL), Golden, CO, USA, 2013.

[9] T. Tsoutsos, N. Frantzeskaki, and V. Gekas, "Environmental impacts from the solar energy technologies," Energy Policy, vol. 33, no. 3, pp. 289-296, 2005.

[10] F. Hyder, K. Sudhakar, and R. Mamat, "Solar PV tree design: a review," Renewable and Sustainable Energy Reviews, vol. 82, pp. 1079-1096, 2018.

[11] H.-M. Neumann, D. Schär, and F. Baumgartner, "The potential of photovoltaic carports to cover the energy demand of road passenger transport," Progress in Photovoltaics: Research and Applications, vol. 20, no. 6, pp. 639-649, 2012.

[12] T. Nordmann and L. Clavadetscher, "PV on noise barriers," Progress in Photovoltaics: research and applications, vol. 12, no. 6, pp. 485-495, 2004.

[13] A. Shekhar, V. K. Kumaravel, S. Klerks et al., "Harvesting roadway solar energy-performance of the installed infrastructure integrated PV bike path," IEEE Journal of Photovoltaics, vol. 8, no. 4, pp. 1066-1073, 2018.

[14] D. Chemisana, "Building integrated concentrating photovoltaics: a review," Renewable and Sustainable Energy Reviews, vol. 15, no. 1, pp. 603-611, 2011.

[15] R. Reisfeld and S. Neuman, "Planar solar energy converter and concentrator based on uranyl-doped glass," Nature, vol. 274, no. 5667, pp. 144-145, 1978.

[16] W. G. J. H. M. van Sark, K. W. J. Barnham, L. H. Slooff et al., "Luminescent solar concentrators - a review of recent results," Optics Express, vol. 16, no. 26, pp. 21773-21792, 2008.

[17] W. G. J. H. M. van Sark, "Luminescent solar concentrators - a low cost photovoltaics alternative," Renewable Energy, vol. 49, pp. 207-210, 2013.

[18] B. C. Rowan, L. R. Wilson, and B. S. Richards, "Advanced material concepts for luminescent solar concentrators," IEEE Journal of Selected Topics in Quantum Electronics, vol. 14, no. 5, pp. 1312-1322, 2008.

[19] C. Corrado, S. W. Leow, M. Osborn et al., "Power generation study of luminescent solar concentrator greenhouse," Journal of Renewable and Sustainable Energy, vol. 8, no. 4, article 043502, 2016.

[20] K. Kapsis and K. Kapsis, Modelling, Design and Experimental Study of Semi-Transparent Photovoltaic Windows for Commercial Building Applications, [Ph.D. thesis], Concordia University, 2016.

[21] F. Meinardi, F. Bruni, and S. Brovelli, "Luminescent solar concentrators for building-integrated photovoltaics," Nature Reviews Materials, vol. 2, no. 12, article 17072, 2017.

[22] F. M. Vossen, M. P. J. Aarts, and M. G. Debije, "Visual performance of red luminescent solar concentrating windows in an office environment," Energy and Buildings, vol. 113, pp. 123-132, 2016.

[23] S. W. Leow, C. Corrado, M. Osborn, and S. A. Carter, "Monte Carlo ray-tracing simulations of luminescent solar concentrators for building integrated photovoltaics," in Proceeding of SPIE 8821, High and Low Concentrator Systems for Solar Electric Applications VIII, 882103, San Diego, CA, USA, 2013.

[24] C. Corrado, S. W. Leow, M. Osborn, E. Chan, B. Balaban, and S. A. Carter, "Optimization of gain and energy conversion 
efficiency using front-facing photovoltaic cell luminescent solar concentratordesign," Solar Energy Materials and Solar Cells, vol. 111, pp. 74-81, 2013.

[25] B. Vishwanathan, A. H. M. E. Reinders, D. K. G. de Boer et al., "A comparison of performance of flat and bent photovoltaic luminescent solar concentrators," Solar Energy, vol. 112, pp. 120-127, 2015.

[26] S. Woei Leow, C. Corrado, M. Osborn, M. Isaacson, G. Alers, and S. A. Carter, "Analyzing luminescent solar concentrators with front-facing photovoltaic cells using weighted Monte Carlo ray tracing," Journal of Applied Physics, vol. 113, no. 21, article 214510, 2013.

[27] X. Sheng, L. Shen, T. Kim et al., "Doubling the power output of bifacial thin-film GaAs solar cells by embedding them in luminescent waveguides," Advanced Energy Materials, vol. 3, no. 8, pp. 991-996, 2013.

[28] J. Yoon, L. Li, A. V. Semichaevsky et al., "Flexible concentrator photovoltaics based on microscale silicon solar cells embedded in luminescent waveguides," Nature Communications, vol. 2, no. 1, p. 343, 2011.

[29] Y. Zhang, S. Sun, R. Kang et al., "Polymethylmethacrylatebased luminescent solar concentrators with bottom-mounted solar cells," Energy Conversion and Management, vol. 95, pp. 187-192, 2015.

[30] J. Zhang, M. Wang, Y. Zhang et al., "Optimization of large-size glass laminated luminescent solar concentrators," Solar Energy, vol. 117, pp. 260-267, 2015.

[31] M. Buffa, S. Carturan, M. G. Debije, A. Quaranta, and G. Maggioni, "Dye-doped polysiloxane rubbers for luminescent solar concentrator systems," Solar Energy Materials and Solar Cells, vol. 103, pp. 114-118, 2012.

[32] K. A. Colby, J. J. Burdett, R. F. Frisbee, L. Zhu, R. J. Dillon, and C. J. Bardeen, "Electronic energy migration on different time scales: concentration dependence of the time-resolved anisotropy and fluorescence quenching of Lumogen Red in poly(methyl methacrylate)," The Journal of Physical Chemistry A, vol. 114, no. 10, pp. 3471-3482, 2010.

[33] G. Maggioni, A. Campagnaro, S. Carturan, and A. Quaranta, "Dye-doped parylene-based thin film materials: application to luminescent solar concentrators," Solar Energy Materials and Solar Cells, vol. 108, pp. 27-37, 2013.

[34] M. E. Loik, S. A. Carter, G. Alers et al., "Wavelength-selective solar photovoltaic systems: powering greenhouses for plant growth at the food-energy-water nexus," Earth's Future, vol. 5, no. 10, pp. 1044-1053, 2017.

[35] J. D. White, "Alternative Energy: Making the controlled environment a greener, more stable place.," Inside Grower, pp. 18-20, 2017.

[36] AZoCleantech, "Solar greenhouses can generate electricity \& grow crops," 2018, https://www.azocleantech.com/news .aspx?newsID=24326.

[37] Z. Cai, W. Qiu, G. Shao, and W. Wang, "A new fabrication method for all-PDMS waveguides," Sensors and Actuators A: Physical, vol. 204, pp. 44-47, 2013.

[38] C. H. Chou, J. K. Chuang, and F. C. Chen, "High-performance flexible waveguiding photovoltaics," Scientific Reports, vol. 3, no. 1, p. 2244, 2013.

[39] C.-H. Chou, M.-H. Hsu, and F.-C. Chen, "Flexible luminescent waveguiding photovoltaics exhibiting strong scattering effects from the dye aggregation," Nano Energy, vol. 15, pp. 729-736, 2015.
[40] S. F. H. Correia, P. P. Lima, E. Pecoraro et al., "Scale up the collection area of luminescent solar concentrators towards metre-length flexible waveguiding photovoltaics," Progress in Photovoltaics: Research and Applications, vol. 24, no. 9, pp. 1178-1193, 2016.

[41] C. Tummeltshammer, A. Taylor, A. J. Kenyon, and I. Papakonstantinou, "Flexible and fluorophore-doped luminescent solar concentrators based on polydimethylsiloxane," Optics Letters, vol. 41, no. 4, pp. 713-716, 2016.

[42] M. Gajic, F. Lisi, N. Kirkwood, T. A. Smith, P. Mulvaney, and G. Rosengarten, "Circular luminescent solar concentrators," Solar Energy, vol. 150, pp. 30-37, 2017.

[43] N. Steinbruck, M. Konemann, and G. Kickelbick, "Effect of polysiloxane encapsulation material compositions on emission behaviour and stabilities of perylene dyes," RSC Advances, vol. 8, no. 32, pp. 18128-18138, 2018.

[44] G. Griffini, M. Levi, and S. Turri, "Thin-film luminescent solar concentrators: a device study towards rational design," Renewable Energy, vol. 78, pp. 288-294, 2015.

[45] M. A. Green, Y. Hishikawa, W. Warta et al., "Solar cell efficiency tables (version 50)," Progress in Photovoltaics: Research and Applications, vol. 25, no. 7, pp. 668-676, 2017.

[46] R. Bose, D. J. Farrell, A. J. Chatten, M. Pravettoni, A. Buchtemann, and K. W. J. Barnham, "Novel configurations of luminescent solar concentrators," in Proceedings of the 22nd European Photovoltaic Solar Energy Conference and Exhibition, pp. 210-214, Milan, 2007.

[47] H. K. Lichtenthaler, "[34] Chlorophylls and carotenoids: pigments of photosynthetic biomembranes," Methods in Enzymology, vol. 148, pp. 350-382, 1987. 

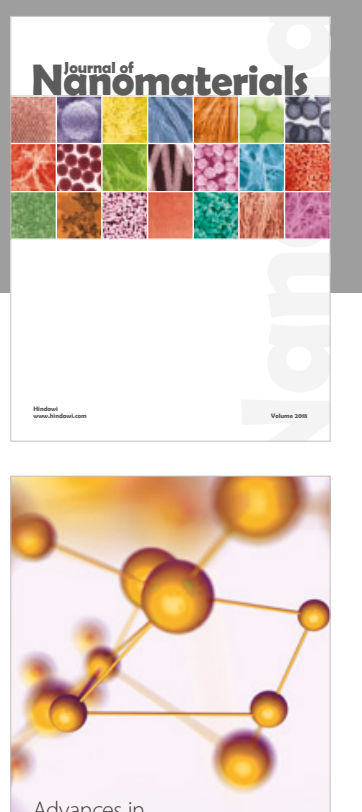

Physical Chemistry
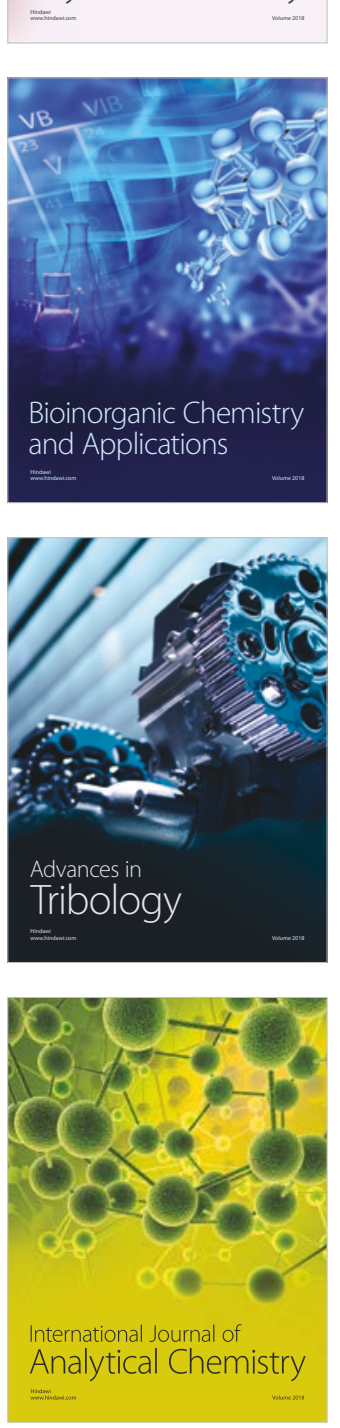

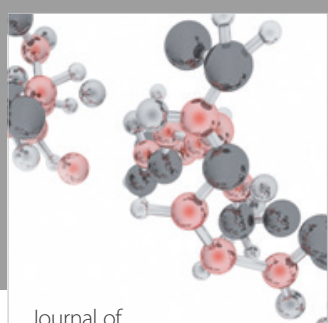

Analytical Methods

in Chemistry

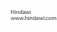

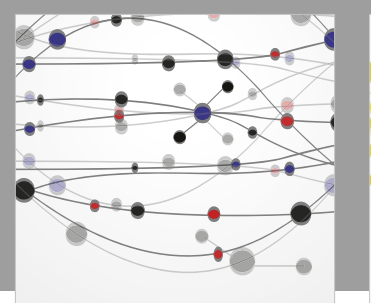

The Scientific World Journal

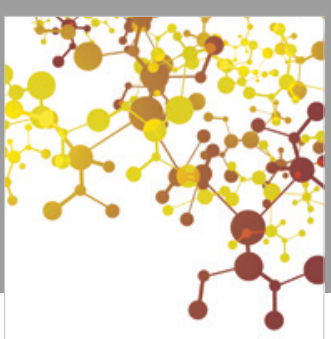

Journal of

Applied Chemistry
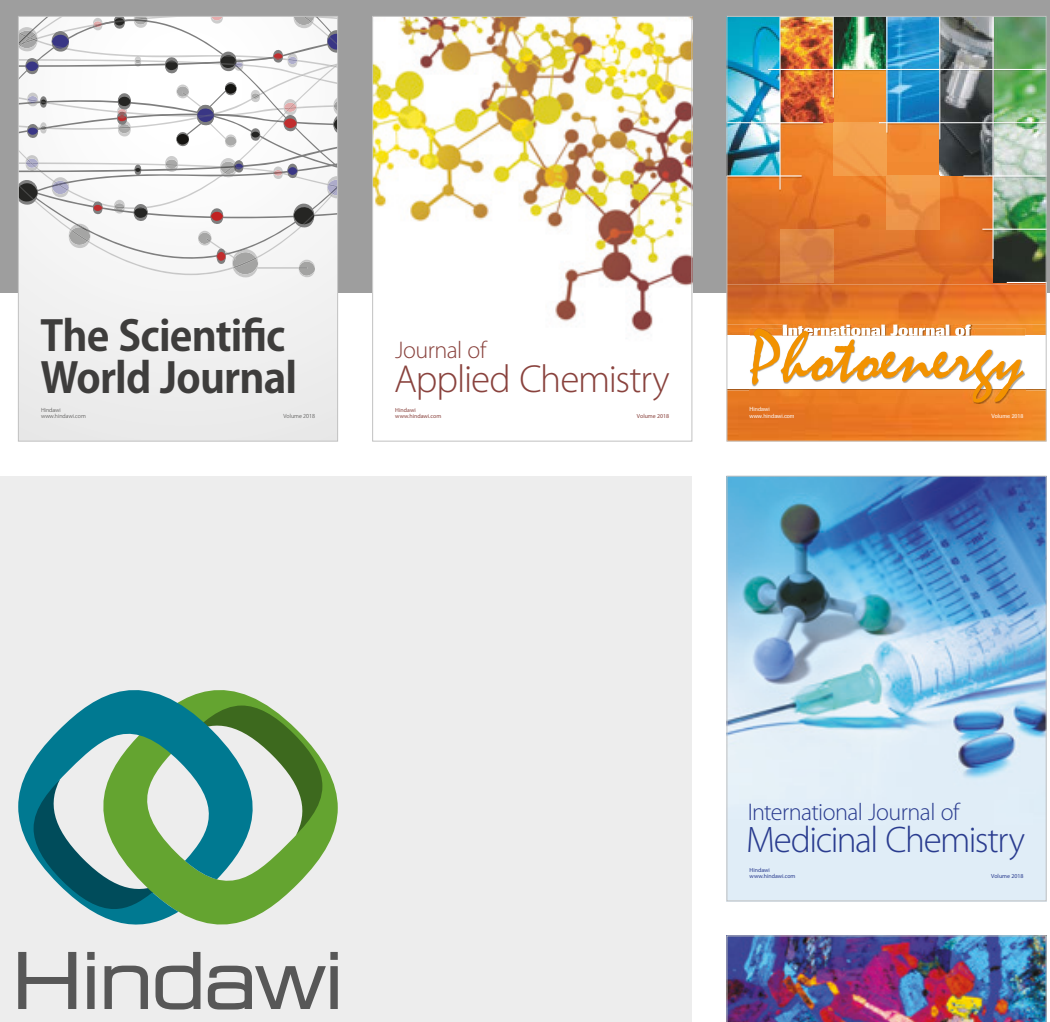

Submit your manuscripts at

www.hindawi.com
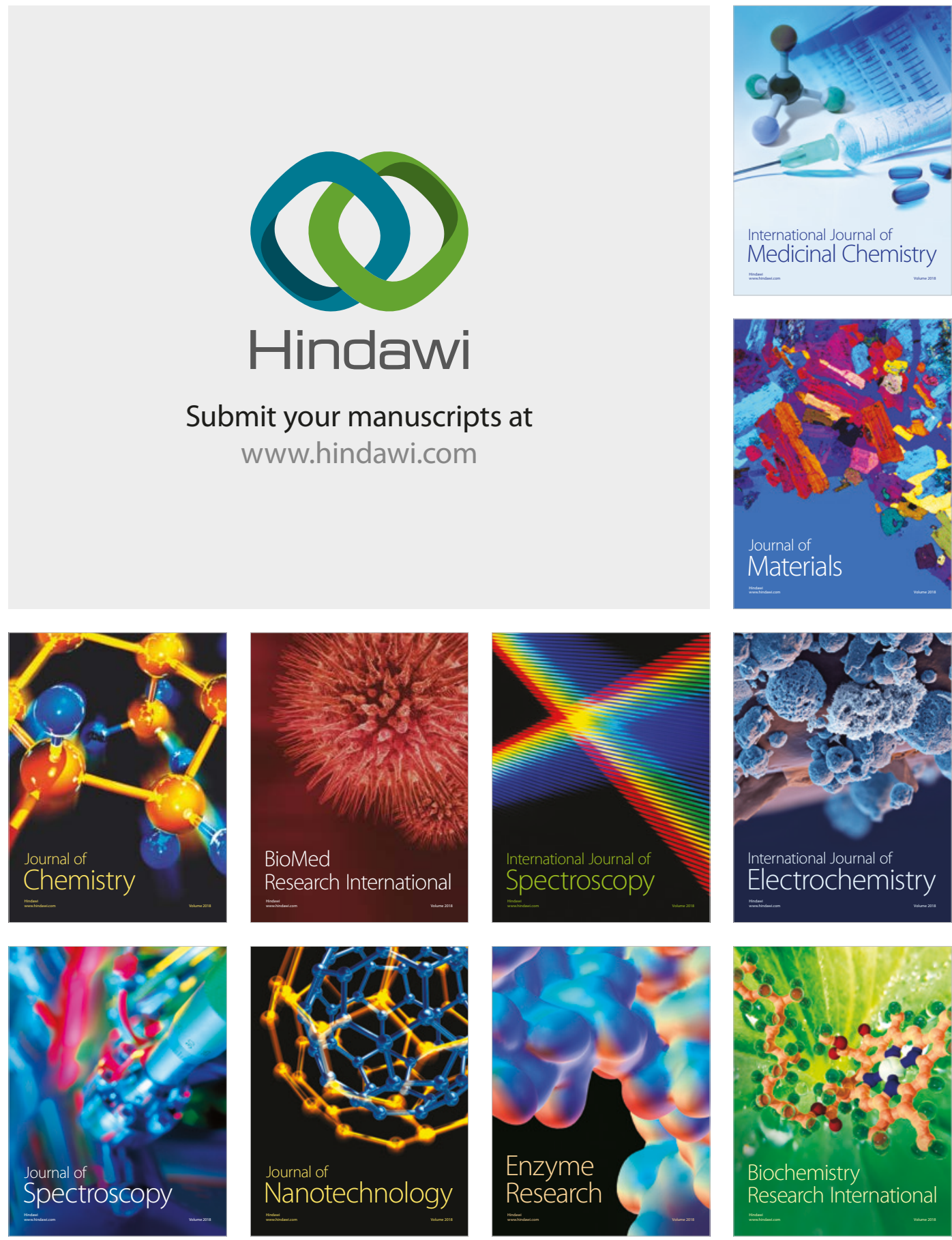
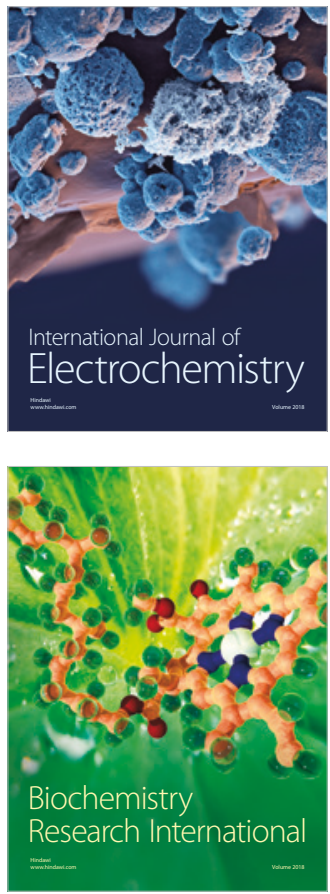